\title{
Deuterium supersaturation in low-energy plasma-loaded tungsten surfaces
}

\author{
L. Gao, W. Jacob*, U. von Toussaint, A. Manhard, M. Balden, K. Schmid and T. Schwarz-Selinger \\ Max-Planck-Institut für Plasmaphysik, Boltzmannstr. 2, 85748 Garching, Germany
}

\begin{abstract}
Fundamental understanding of hydrogen-metal interaction is challenging due to lack of knowledge on defect production and/or evolution upon hydrogen ingression, especially for metals undergoing hydrogen irradiation with ion energy below the reported displacement thresholds from literature. Here, applying a novel low-energy argon-sputter depth-profiling method with significantly improved depth resolution for tungsten (W) surfaces exposed to deuterium (D) plasma at $300 \mathrm{~K}$, we show the existence of a 10-nm-thick D-supersaturated surface layer (DSSL) with an unexpectedly high D concentration of $\sim 10$ at. $\%$ after irradiation with ion energy of $215 \mathrm{eV}$. Electron back-scatter diffraction reveals that the W lattice within this DSSL is highly distorted thus strongly blurring the Kikuchi pattern. We explain the strong damage by the synergistic interaction of the energetic $\mathrm{D}$ ions and solute $\mathrm{D}$ atoms with the $\mathrm{W}$ lattice. Solute $\mathrm{D}$ atoms prevent the recombination of vacancies with interstitial $\mathrm{W}$ atoms, which are produced by the collisions of energetic $\mathrm{D}$ ions with W lattice atoms (Frenkel pairs). This proposed damaging mechanism could also be active on other hydrogen-irradiated metal surfaces. The present work provides a deep insight into hydrogen-induced lattice distortion at plasma-metal interfaces and sheds light on its modelling work.
\end{abstract}

Keywords: Plasma-surface interaction; Hydrogen-induced damage; Solute hydrogen; Hydrogen decoration; Nuclear reaction analysis.

PACS numbers: 52.40.Hf, 52.77.Dq, 68.43.-h, 79.20.Rf

$\begin{array}{ll}\text { Published in: } & \text { Nuclear Fusion } 57 \text { (2017) } 016026 \text { (11pp). } \\ \text { doi: } & 10.1088 / 0029-5515 / 57 / 1 / 016026\end{array}$

Submitted: $\quad 09.09 .2016$

Accepted: $\quad 04.10 .2016$

Available online: 11.11.2016

\footnotetext{
${ }^{*}$ Corresponding author. Fax: +49-3299-1504, Tel: +49-3299-2618.

E-mail address: wolfgang.jacob@ipp.mpg.de
} 


\section{Introduction}

Hydrogen $(\mathrm{H})$ interacts strongly with almost all solid materials and modifies their general properties. E.g., metal hydride formation has been extensively investigated for $\mathrm{H}$ fuel storage [1-3]. Furthermore, $\mathrm{H}$ embrittlement [4- 6] is expected as one major reason for environmental degradation and enhances the tendency for fracturing and cracking in structural alloys. The Hmetal interaction is in general very complex [7] regardless of how $\mathrm{H}$ ingresses into the material. However, due to the lack of knowledge on defect production and/or evolution upon $\mathrm{H}$ ingression, many aspects of this phenomenon are still poorly understood in spite of more than a century of studies. Recently, the planned use of tungsten (W) as plasma-facing material (PFM) for the divertor region of the international thermonuclear fusion experiment ITER $[8,9]$ and next-generation devices has triggered particular attention on the interaction of hydrogen isotopes plasmas with $\mathrm{W}$ surfaces. One of the relevant favorable properties of $\mathrm{W}$ for the operation of a future fusion device is the extremely low solubility for hydrogen isotopes, such as the valuable and radioactive - and therefore hazardous - fusion fuel tritium [10, 11]. On one hand, this low hydrogen solubility means that the potential hydrogen isotopes retention in $\mathrm{W}$ is practically exclusively determined by the types and densities of defects in the material $[10,11]$, which act as "traps" for hydrogen isotopes. Such traps can either be intrinsic, i.e. already being present in the material for example due to industrial processing or impurities, be produced by neutron-irradiation-induced damage from the high energetic fusion neutrons during D-T fusion plasma operation (see, e.g., Refs. $[12,13]$ and references therein), or such defects can be hydrogen-induced, i.e. being produced during loading in hydrogen plasma. On the other hand, the strong endothermic heat of solution of hydrogen in W together with the fact that $\mathrm{W}$ does not form hydrides can lead to an extreme chemical potential due to the oversaturation of $\mathrm{W}$ with solute hydrogen. Such an extremely non-equilibrium state is difficult to achieve in most cases, but it can be relatively easily reached in plasma-irradiated samples. The high oversaturation may facilitate near-surface modifications, e.g. by damage production even below the energy threshold for displacement damage thus modifying the final hydrogen retention in $\mathrm{W}$ materials. In other words, hydrogen plasma exposure of $\mathrm{W}$ materials could lead to strongly damaged surfaces and in turn high hydrogen retention, as it will be shown in the present work.

One key method to understand the plasma-surface-interaction process is $\mathrm{H}$ depth profiling after loading. Compared with other elements $\mathrm{H}$ behaves rather elusive in standard surface analysis, because it can only be directly detected by a few techniques such as nuclear reaction analysis (NRA) via resonant ${ }^{1} \mathrm{H}\left({ }^{15} \mathrm{~N}, \alpha \gamma\right){ }^{12} \mathrm{C}$ reaction $\left({ }^{15} \mathrm{~N} \mathrm{NRA}\right)$ [14] for $\mathrm{H}$ or via $\mathrm{D}\left({ }^{3} \mathrm{He}, \mathrm{p}_{0}\right) \propto$ reaction $\left({ }^{3} \mathrm{He} \mathrm{NRA}\right)[15,16]$ for deuterium (D), and thermal desorption spectroscopy (TDS) [17]. ${ }^{15} \mathrm{~N}$ NRA is widely applied in detecting $\mathrm{H}$ at solid surfaces and interfaces with very high depth resolution. However, in many cases $\mathrm{H}$ retention is studied by loading the samples with $\mathrm{D}$ and measuring the $\mathrm{D}$ inventory and depth distribution using ${ }^{3} \mathrm{He}$ NRA, in particular for the investigation of $\mathrm{H}$ isotopes in some transition metals with extremely low $\mathrm{H}$ solubility, e.g., $\mathrm{W}$ and iron, because it is rather difficult to distinguish the retained $\mathrm{H}$ in such metals from the ubiquitous background $\mathrm{H}$ and from surface adsorbed $\mathrm{H}$ (e.g. in form of adsorbed water) using ${ }^{15} \mathrm{~N}$ NRA. In addition, ${ }^{3} \mathrm{He} \mathrm{NRA}$ is for some applications more favorable due to a larger information depth compared with ${ }^{15} \mathrm{~N}$ NRA. D depth profiles in plasma-exposed $\mathrm{W}$ surfaces determined by deconvolving the acquired proton and alpha spectra from ${ }^{3} \mathrm{He}$ NRA measurements using the NRADC data evaluation program [16] usually show the existence of a thin D-rich layer directly at the topmost W surface [1720]. However, the depth resolution of the commonly used ${ }^{3} \mathrm{He}$ NRA depth profiling method is insufficient to properly resolve this D-rich layer. For our experimental set-up the best achievable depth resolution at the surface using a ${ }^{3} \mathrm{He}^{+}$projectile energy of $690 \mathrm{keV}$ is 
$\sim 16 \mathrm{~nm}$. This depth resolution is based on the analysis of the produced $\alpha$ particles $[15,16]$. As a consequence, NRADC can only provide an averaged D concentration for the topmost $16 \mathrm{~nm}$. Applying ${ }^{3} \mathrm{He}$ NRA followed by NRADC data evaluation D concentrations in the topmost layer of about 3-4 at.\% were reported [17-20]. In the present work, applying lowenergy argon sputtering followed by ion beam analysis (IBA) - a newly-developed D depthprofiling method from our previous work [21,22] - we first resolve this D-rich surface layer in plasma-exposed $\mathrm{W}$ with a significantly improved depth resolution of about $3 \mathrm{~nm}$. We will show that the D-rich layer is substantially thinner than $16 \mathrm{~nm}$ and that the actual D concentrations in it are significantly higher. Because of these - for pristine crystalline $\mathrm{W}-$ extreme D concentrations we will in the following call this layer "D-supersaturated surface layer (DSSL)". Subsequently, we apply high-resolution scanning electron microscopy (SEM) and electron back-scatter diffraction (EBSD) to study the structural changes induced in the surface due to the evolution of the DSSL. Finally we propose a novel damaging mechanism occurring in the DSSL during plasma loading.

\section{Experimental details}

\subsection{D plasma exposure}

Poly-crystalline, hot-rolled W samples $\left(15 \times 12 \times 0.7 \mathrm{~mm}^{3}, \quad 99.97\right.$ wt. \% purity, manufactured by Plansee SE, Austria) were chemo-mechanically polished to a mirror-like finish [23] and annealed in vacuum at $1200 \mathrm{~K}$ for 2 hours. D implantations were performed in a quantified plasma source [24]. At the operating pressure of $1.0 \mathrm{~Pa}$ this plasma source delivers an ion flux of primarily $\mathrm{D}_{3}{ }^{+}$ions $(94 \%)$ with minor contributions of $\mathrm{D}_{2}{ }^{+}(3 \%)$ and $\mathrm{D}^{+}$ (3\%). The chosen ion bombardment energy of $\sim 215 \mathrm{eV}$ corresponds to a mean energy of $72 \mathrm{eV}$ per $\mathrm{D}$ for the dominant ion species $\mathrm{D}_{3}{ }^{+}$. The used deuteron flux was $9.9 \times 10^{19} \mathrm{D} \mathrm{m}^{-2} \mathrm{~s}^{-1}$ [24]. It took roughly 17 hours for accumulating the chosen D fluence of $6 \times 10^{24} \mathrm{D} \mathrm{m}^{-2}$. The sample temperature was stabilized at $300 \mathrm{~K}$ using an ethanol cooling circuit with a thermocouple integrated in the substrate holder just underneath the samples [24].

\subsection{Ion beam analysis and argon-sputter depth profiling}

${ }^{3} \mathrm{He}$ NRA was applied for measuring the retained D amounts in $\mathrm{W}$ samples after D implantation. All D-implanted W samples were measured with NRA applying 7 different ${ }^{3} \mathrm{He}^{+}$ projectile energies ranging from 0.5 to $4.5 \mathrm{MeV}$. The produced high energy protons from NRA were counted using a thick, large angle solid state detector at a scattering angle of $135^{\circ}$ equipped with a curved slit reducing the solid angle to $29.9 \mathrm{msr}$. The produced $\alpha$ particles were counted using a surface barrier detector at the laboratory scattering angle of $102^{\circ}$ equipped with a rectangular slit reducing the solid angle to $9.16 \mathrm{msr}$. For each individual NRA spectrum an ion beam charge of $10 \mu \mathrm{C}$ was accumulated. The information depth for the NRA analysis is defined as the depth where the kinetic energy of the projectiles decreased to about $420 \mathrm{keV}$ (the energy where the cross-section has decreased to $10 \%$ of the maximum value at $620 \mathrm{keV}$ [15]) for the present nuclear reaction. This information depth was calculated applying SIMNRA [25] with Ziegler/Biersack stopping power data, which results in a value of $\sim 7.9 \mu \mathrm{m}$ for the maximum ${ }^{3} \mathrm{He}$ projectile energy of $4.5 \mathrm{MeV}[17,26]$. The generated protons have a very high energy of about $13.5 \mathrm{MeV}$. The mean free path of these high energy protons is much larger than this information depth, such that the generated protons can easily reach the detector. For $690 \mathrm{keV}$ ion beam exhibiting the highest cross-section among all the applied 7 projectile energies, the ${ }^{3} \mathrm{He}$ NRA information depth in $\mathrm{W}$ is about $800 \mathrm{~nm}$ for the high energy protons. For the given experimental geometry, the corresponding depth resolution from protons at the surface is $110 \mathrm{~nm}$ according to RESOLNRA [25]. The depth resolution from the analysis of $\alpha$ particle spectra is better due to the much stronger stopping by the 
material than for the high-energy protons. For our experimental set-up the best achievable depth resolution at the surface using a ${ }^{3} \mathrm{He}^{+}$projectile energy of $690 \mathrm{keV}$ is $\sim 16 \mathrm{~nm}[16,17]$. However, it turns out that even with this improved depth resolution from the analysis of the $\alpha$ particle spectra, the commonly used NRA depth profiling method is still insufficient to properly resolve the DSSL. We, therefore, applied the newly-developed Ar-sputter IBA depth profiling method $[21,22]$ to resolve this DSSL.

After D implantation W samples were exposed to argon plasma in the same plasma device as for D plasma exposure applying an Ar pressure of $0.5 \mathrm{~Pa}$ and a DC bias voltage of $-200 \mathrm{~V}$. Ar plasma exposure was performed for different durations on different sections of two identical samples by partially covering the sample surface with a $50 \mu \mathrm{m}$ thick $\mathrm{W}$ foil while keeping other parts exposed to Ar plasma. During Ar sputtering the sample temperature was kept at $230 \mathrm{~K}$ using the ethanol cooling circuit to inhibit a possible D loss due to surface heating by the plasma as well as D diffusion into the bulk during Ar sputtering. Before applying the DC bias, all samples were exposed to Ar plasma at floating potential $(3 \mathrm{~V})$ for 10 min to gently remove the surface adsorbed contamination. In total, six different sections from two identical $\mathrm{W}$ samples each about $5 \times 6 \mathrm{~mm}^{2}$ in area with sputtering time from 0 to 25 min with 5-min intervals were created. This strategy allows us to measure the remaining D amount in the 6 different sections in one experimental session with the same ${ }^{3} \mathrm{He}^{+}$ion beam for the remaining D amount by NRA. Because Ar sputtering was conducted on different areas of the sample surfaces it has first to be checked that D retention in different samples or on different areas of the same sample is similar, such that the NRA measurements from different samples or areas can later be compared. Therefore, in independent experiments we compared $\mathrm{D}$ retention in different samples and the lateral homogeneity of the $\mathrm{D}$ depth profiles on the same sample for loading conditions as given above. It turned out that D depth profiles measured in different areas of our samples are well comparable. This allows us to directly compare the $\alpha$ and proton spectra from sample surfaces with different Ar sputtering durations measured with identical ${ }^{3} \mathrm{He}$ ion beam.

From ${ }^{3} \mathrm{He}$ NRA we can measure the remaining D amount in the sample after each Ar sputtering step. To determine the D concentration, the amount of $\mathrm{W}$ being removed by each step has to be measured. This is achieved by exposing a thin W film (100 nm thick, sputterdeposited on single-crystalline Si substrate) simultaneously with the bulk W sample to the identical Ar plasma during each step. The areal density and the precise thickness of the thin W film were determined prior to Ar sputtering by Rutherford Back-scattering Spectroscopy and profilometry, respectively. The atomic density of the deposited $\mathrm{W}$ films has been determined before $[17,27]$, which is roughly $90 \%$ of the bulk W density of $19.3 \mathrm{~g} / \mathrm{cm}^{3}$. The thickness of the removed layer by the different Ar sputtering durations was then determined. It turned out that a 5-min Ar sputtering step can remove a thickness of $3.4 \pm 0.2 \mathrm{~nm}$ of the $\mathrm{W}$ film. It is assumed that the sputtering yield is the same for the $\mathrm{W}$ film and bulk W surface, so the removed thickness of bulk $\mathrm{W}$ is expected to be $3.1 \pm 0.2 \mathrm{~nm}$ due to its slightly higher atomic density. It should be noted that with a shorter Ar-sputtering step the depth resolution of the present method can in principle be improved such that a depth resolution of about $1 \mathrm{~nm}$ should be achievable.

For comparison and reference an about $10 \mathrm{~nm}$ thick plasma-deposited amorphous deuterated carbon film (a-C:D) on a Si wafer was also measured with the same ${ }^{3} \mathrm{He}$ ion beam as the D-implanted $\mathrm{W}$ surfaces. The D amount in the sample was quantified using the crosssection data published by Alimov et al. [15]. This results in a D amount of $(5.6 \pm 0.1) \times 10^{20} \mathrm{D}$ atoms $/ \mathrm{m}^{2}$ in the a-C:D layer. The thickness of this a-C:D layer is thinner than the best achievable depth resolution for a-C:D (which is about $13 \mathrm{~nm}$ as calculated by 
RESOLNRA [25]). Therefore, both the $\alpha$ particle and proton spectrum from the a-C:D layer exhibit a narrow surface peak with a peak width, which is determined only by the experimental broadening. We assume the same broadening effect for the DSSL on our W samples such that the peak widths of the $\alpha$ particle and proton spectra of the DSSL should be comparable to those from the a-C:D layer. The resulting peak shape of our D-implanted W samples is then comprised of the surface peak and of contributions from D retained in larger depth. Because the thickness of the DSSL is much thinner than the information depth at this energy its removal does not influence the bulk contribution to the NRA spectra. The bulk contribution is further assumed to be determined by the peak integrals for Ar sputtering times longer than 20 min (see Fig. 2). We then determine the contribution of the DSSL to the NRA spectra by subtracting this bulk contribution for each sputtering step.

\subsection{SDTrimSP simulation}

SDTrimSP $[28,29]$ simulations of D implantation were performed to interpret the present experimental results. Based on the TRIDYN code [28], SDTrimSP [29] and related simulation tools describe the transport of energetic ions in matter on the basis of the binary collision approximation. The simulations were performed for normal impact angle and an energy distribution corresponding to a $\mathrm{D}$ flux consisting predominantly of $\mathrm{D}_{3}{ }^{+}$ions $(94 \%)$ and minority contributions of $\mathrm{D}^{+}(3 \%)$ and $\mathrm{D}_{2}{ }^{+}$ions $(3 \%)$, which is identical to the ion species distribution of D plasma generated in the present plasma device [24]. The molecular ions are treated as individual deuterons impinging with the same velocity. For example, a $\mathrm{D}_{3}{ }^{+}$ion with energy of $215 \mathrm{eV}$, as in the present case, will be treated as three impinging deuterons with energy of $72 \mathrm{eV}$ and a $\mathrm{D}_{2}^{+}$corresponds to two deuterons with energy of $108 \mathrm{eV}$. Here, SDTrimSP simulations are employed to calculate the energy transfer in D collision with the W lattice atoms as a function of depth. SDTrimSP was additionally applied to calculate the ion range of impurity ions (e.g., C, N, O, Ar) with fixed ion energy of $215 \mathrm{eV}$. The simulations were performed in static mode, that means the possible retention of impurity ions in the matrix and its influence on stopping was not taken into account, or with other words each impurity ion impinged always on the pure $\mathrm{W}$ matrix.

\subsection{Degassing}

The degassing experiments were performed in a high-vacuum oven where the hot zone, heated by radiation, is constructed entirely from molybdenum. After pumping for 6 hours, the base pressure before heating was typically lower than $3 \times 10^{-5} \mathrm{~Pa}$. At the annealing temperature the vacuum was still better than $10^{-3} \mathrm{~Pa}$. The specimens were held at the set temperatures for $10 \mathrm{~min}$. After this holding time, the specimens were cooled to room temperature as quickly as possible. Details of outgassing slightly depend on loading conditions and sample preparation, but the vast majority (more than $90 \%$ ) of $\mathrm{D}$ is desorbed at $\mathrm{T} \leq 750 \mathrm{~K}[30,31]$.

\section{Results and discussion}

W surfaces after D implantation at $300 \mathrm{~K}$ with an ion energy of $215 \mathrm{eV}$ were exposed to Ar plasma for different durations and then measured with ${ }^{3} \mathrm{He}$ NRA for D depth profiling [16, 17]. Fig. 1 shows the $\alpha$ and proton raw spectra from the NRA measurements with a $690 \mathrm{keV}^{3} \mathrm{He}^{+}$beam of D-implanted W surfaces with Ar sputtering times ranging from 0 to 25 min with 5-min intervals (see Sec. 2.2). Also shown as dashed line is the spectrum of a thin a-C:D layer with a D amount of $5.6 \times 10^{20} \mathrm{D} / \mathrm{m}^{2}$ (see Sec. 2.2) which is used to determine the apparatus function for the detection of the NRA reaction products. This a-C:D layer shows an $\alpha$ particle spectrum with a narrow surface peak only. This peak is located at about $4.8 \mathrm{MeV}$. 
In contrast, the $\alpha$ particle spectra of D-implanted W surfaces (Fig. 1a) exhibit similar surface peaks and additionally small contributions at lower energy $(2-4.6 \mathrm{MeV})$, which are attributed to $\mathrm{D}$ in larger depth. Already after the first Ar-sputtering step the $\alpha$ surface peak has significantly decreased and it has almost disappeared after 3 sputtering steps (i.e. after 15 min Ar sputtering). The $\alpha$ signals from larger depth are practically unchanged for all the measured surfaces. Also the proton peak (Fig. 1b) decreases significantly after sputtering. The difference between the third and fourth sputtering step is very small and after the fourth sputtering step, both $\alpha$ and proton spectra do not change anymore. By this, we attribute the disappearance of the $\alpha$ surface peak as well as the decrease of the proton peak to the removal of the DSSL by Ar sputtering. The proton counts remaining in the energy range from 12.9 to 13.2 MeV after 3 sputtering steps (Fig. 1b) are due to nuclear reactions with D in larger depths (i.e. beyond $\sim 9 \mathrm{~nm}$ since each 5 min Ar-sputtering step removes $\sim 3 \mathrm{~nm} \mathrm{~W}$ material, see in Sec. 2.2). They contribute to the signal in this energy range due to geometrical straggling [26], which was confirmed by SIMNRA simulations [25].
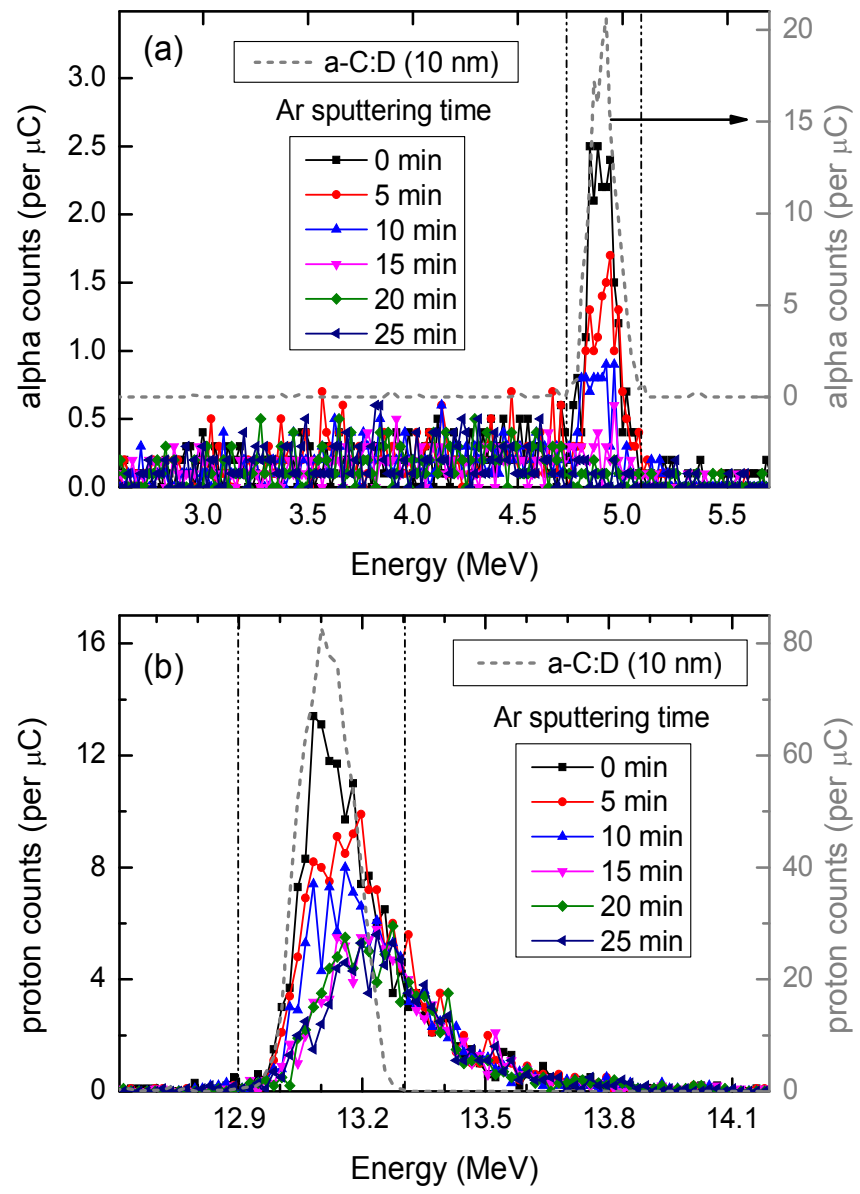

Figure 1. (color online only) Raw spectra from NRA measurements with $0.69 \mathrm{MeV}^{3} \mathrm{He}^{+}$beam after different Ar sputtering durations: a) a spectra; b) proton spectra. The $D$ implantation in $W$ was performed at $300 \mathrm{~K}$ with $215 \mathrm{eV}$ ion energy to a fluence of $6 \times 10^{24} \mathrm{D} \mathrm{m}^{-2}$. The dashed line in both figures shows the spectrum from an about $10 \mathrm{~nm}$ thick a-C:D layer (right-hand $y$ scale). The vertical dash-dotted lines indicate the energy range for the integration applied in the following data evaluation (see data in Fig. 2).

The integrals of the raw spectra from Fig. 1 for the energy range marked by the 2 vertical dash-dotted lines (i.e. taken from the footprints of the thin a-C:D layer) are plotted in Fig. 2a as a function of the Ar sputtering time. These peak integrals are correlated to the total amount of $\mathrm{D}$ in the topmost layer. The error bars are evaluated based on the counting statistics (i.e. 
square root of the counts). Both proton and $\alpha$ integrals decrease significantly for the first 3 sputtering steps and remain essentially constant afterwards. Actually, the proton integrals measured at other projectile energies (not shown) exhibit the same decreasing behavior. Already from these raw data one can conclude that a substantial fraction of the D was retained in the removed $\sim 9 \mathrm{~nm}$ thick $\mathrm{W}$ surface layer, which is much thinner than the NRA information depth for protons for $690 \mathrm{keV}(\sim 800 \mathrm{~nm})$. We assume that the bulk contribution to the proton signal (i.e. the signal from the remaining $\sim 800 \mathrm{~nm}$ after removal of the DSSL) is given by the average value of the last two corresponding data points in Fig. 2a. This average value is marked by the horizontal line in Fig. 2a. This assumption is justified because the energy loss of the impinging projectiles within the DSSL is negligible, such that the cross section for the detection of the D bulk contributions remains unchanged. Subtracting this bulk $\mathrm{D}$ contribution from the integral values results in the DSSL contributions shown in Fig. 2b. A similar strategy is also applied for the $\alpha$ integrals. The error bars in Fig. $2 b$ are determined according to error propagation due to the background subtraction. Taking into account the error bars for each data point, we fit the first 4 data points for both $\alpha$ and proton integrals. Within the experimental uncertainty both data sets can be well described by a linear decrease. This indicates a uniform D distribution in the DSSL, which is removed by the first 3 to 4 sputter steps followed by a relative sharp decrease of the D concentration. The precise thickness of the DSSL is determined from the intersection of the linear fits with the $\mathrm{x}$ axis. According to these linear fits it takes $(16.8 \pm 0.5)$ min Ar plasma exposure to erode the whole DSSL, which corresponds to a thickness of $(10.4 \pm 0.4) \mathrm{nm}$. The total retained D amount in the DSSL is $(6.8 \pm 0.5) \times 10^{19} \mathrm{D} / \mathrm{m}^{2}$ determined based on the experimentally measured crosssection data from Alimov et al. [15]. Note that this D amount contained in the DSSL is about $30 \%$ of the total retained D amount in the whole NRA information depth $\left(\sim 7.9 \mu \mathrm{m}\right.$ at ${ }^{3} \mathrm{He}$ projectile energy of $4.5 \mathrm{MeV}$ [26]). We further want to emphasize that this D amount cannot be explained by surface adsorption as observed with in-situ experiments on W single crystals by Tamm and Schmidt [32] and on W poly crystals by Markelj et al. [33]. Such an assignment contradicts the experimentally observed homogeneous distribution over the $9 \mathrm{~nm}$ thick W layer. The D concentration in the DSSL calculated from the total amount and the DSSL thickness is $(9.4 \pm 0.7)$ at. \%. Such an extremely high D concentration in crystalline W material after low-energy D plasma loading has not yet been observed before. It is more than three orders of magnitude higher than the maximum steady-state solute D concentration at the D implantation depth $\left(10^{-3}\right.$ at. \%) (see details in APPENDIX) calculated based on an analytical 1-dimensional flux-balance model $[34,35]$ using Frauenfelder's H diffusivity in W [36]. Obviously, a presently unknown process between the implanted D and the W lattice is active in the formation of the DSSL during plasma loading. 


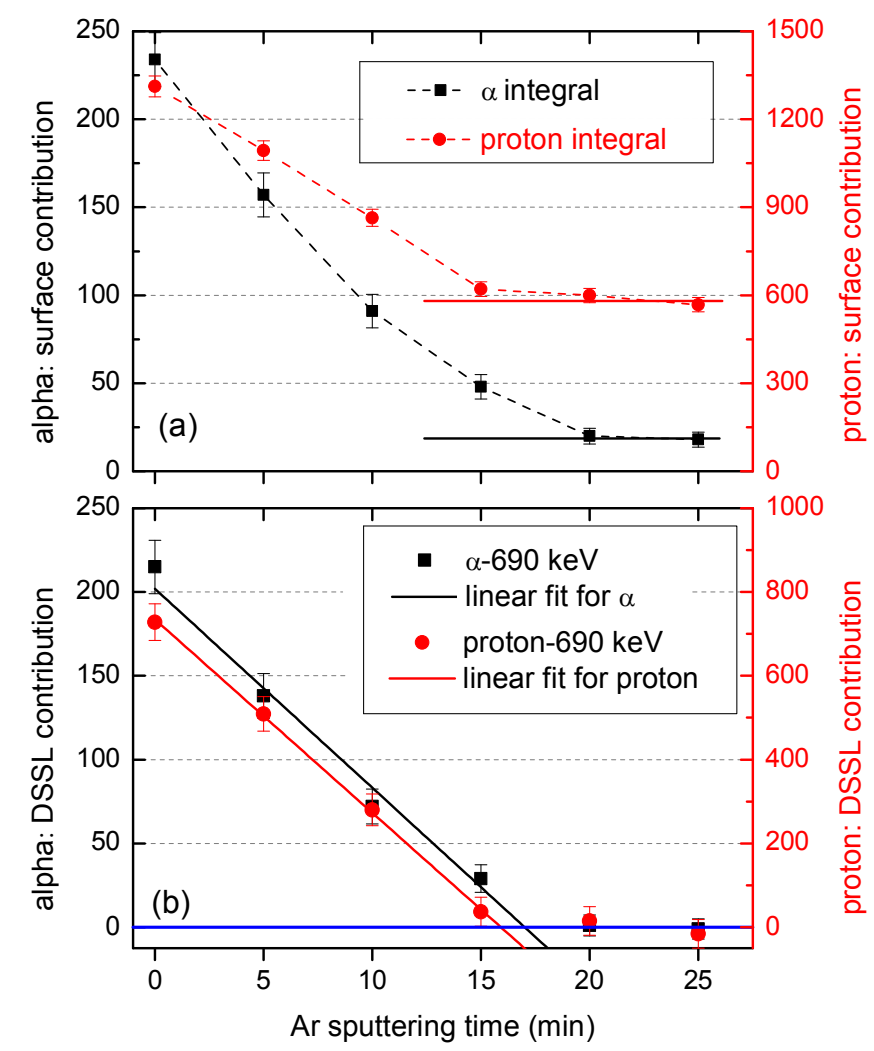

Figure 2. (color online only) Count integrals of $\alpha$ and proton spectra for the energy range marked by the vertical dash-dotted lines in Fig. 1 (i.e. taken from the footprints of the thin a-C:D layer) as a function of Ar sputtering durations: a) $\alpha$ and proton contributions from sample surfaces; $b$ ) contributions only from the DSSL by subtracting the bulk contributions (= average value of the last two data points, marked by horizontal solid lines in (a)). Different y scales apply for $\alpha$ and proton spectra. The error bars in a) are evaluated based on the counting statistics (i.e. square root of the counts) and in b) according to error propagation due to the background subtraction.

For $\mathrm{W}$ exhibiting such a high $\mathrm{D}$ concentration the only reasonable explanation is that the $\mathrm{W}$ material at the topmost surface after D plasma loading is highly distorted (damaged). In principle, the surface could get damaged during the sample preparation before irradiation. However, it was shown before and confirmed here (Fig. 3a) that our sample preparation process [23] produces $\mathrm{W}$ surfaces without visible distortion and gives rise to clear Kikuchi patterns [37]. On the contrary, EBSD measurements after D implantation show very blurred Kikuchi patterns. This indicates that the highly-distorted near-surface region in $\mathrm{W}$ is produced during D implantation. This surface distortion is even visible in SEM images with backscatter electron contrast (see in Fig. 3c). More interestingly, EBSD measurements performed on Dimplanted W surfaces after different Ar sputtering fluences reveal that for W surfaces with less than $15 \mathrm{~min}$ Ar sputtering (i.e. before completely removing the DSSL) the Kikuchi pattern is still blurred, while once the DSSL is removed the Kikuchi pattern as well as the SEM image become clear again (compare Figs. 3c and 3d). The fact that the Kikuchi patterns are observable after extended Ar sputtering (e.g., $15 \mathrm{~min}$ ) proves that the damage produced by Ar ions during sputtering is not sufficient to significantly blur the Kikuchi pattern. This is independently confirmed by exposing a virgin W sample to the same Ar plasma for 15 min (Fig. 3b). We cannot definitely exclude that during D implantation some impurities in the plasma (e.g., oxygen ions from water or nitrogen from background gas) cause some surface damage. However, the penetration depths for $\mathrm{Ar}, \mathrm{N}$ and $\mathrm{O}$ ions impinging on a W surface with energy of $215 \mathrm{eV}$ calculated using SDTrimSP [28, 29] are 1.1, 1.6 and $1.5 \mathrm{~nm}$, respectively. By making the very reasonable assumption that the possible damage produced by impurity 
ions is restricted to the ion penetration range (i.e. 1-2 nm) we conclude that the highlydistorted W layer that coincides with the DSSL cannot be explained by impurity ion implantation. But, more importantly, it can neither be explained by simple displacement damage induced by the impinging $\mathrm{D}$ ions. At $215 \mathrm{eV}, \mathrm{D}$ can at most transfer $9.2 \mathrm{eV}$ to $\mathrm{W}$ target atoms by momentum exchange. The reported displacement threshold energies for $\mathrm{W}$ in the bulk range from 42 to $70 \mathrm{eV}[38,39]$. This means that $\mathrm{D}$ ions with $215 \mathrm{eV}$ cannot create displacement damage in bulk W. Consequently, the observed damage has to be induced by D through another process.
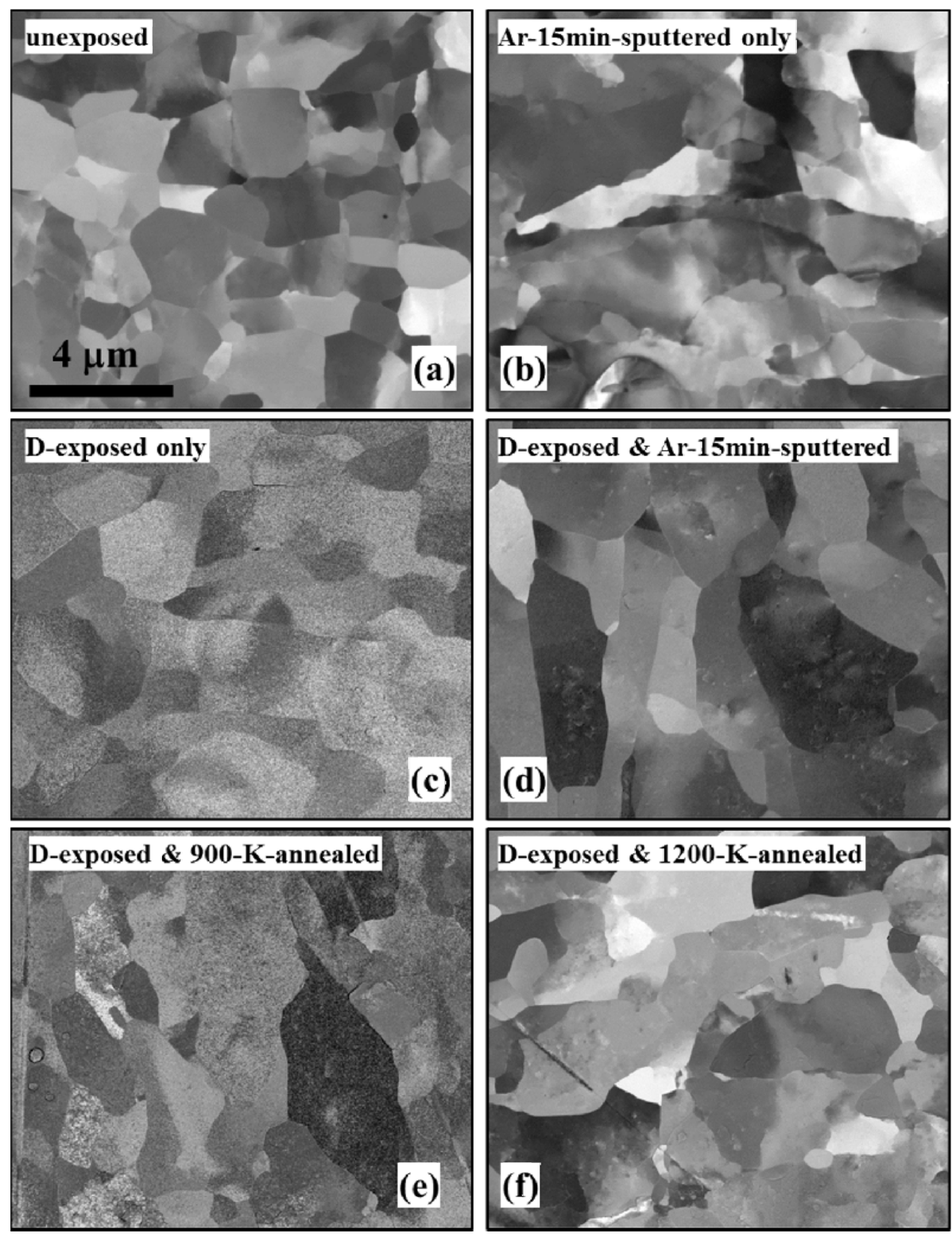

Figure 3. SEM images (produced using $5 \mathrm{keV}$ electrons) of $W$ surfaces after different treatments: a) as-prepared $\mathrm{W}$ surface prior to D implantation (with $2 \mathrm{~h}$ annealing at $1200 \mathrm{~K}$ ); b) after $15 \mathrm{~min} \mathrm{Ar}$ sputtering only; c) after D implantation to fluence of $6 \times 10^{24} \mathrm{D} / \mathrm{m}^{2}$ at $300 \mathrm{~K}$; d) D-implantation followed by $15 \mathrm{~min}$ Ar sputtering (i.e. after removal of the DSSL). e) D-implantation followed by 10 min annealing at $900 \mathrm{~K}$ (i.e. after D degassing); f) D-implantation followed by 10 min annealing at $1200 \mathrm{~K}$. Strong surface distortion is induced by D implantation and can be removed by Ar sputtering or by annealing at temperatures $\geq 1200 \mathrm{~K}$. 
One could argue that the high solute D concentration in the ion implantation range $\left(\sim 2 \times 10^{-5}\right.$, see in APPENDIX) is sufficient to produce the observed damage, but due to the relatively high diffusivity of $\mathrm{D}$ in $\mathrm{W}$ this solute $\mathrm{D}$ concentration is spread over much larger distances (i.e. several $\mu \mathrm{m}$, see details in APPENDIX) than the observed thickness of the DSSL. We, therefore, postulate that the damage in the DSSL is produced by the synergistic interaction of a solute D concentration far above the thermodynamic equilibrium solubility and energetic $\mathrm{D}$ atoms with the $\mathrm{W}$ lattice. For this process to occur still a sufficiently large amount of energy has to be transferred to a $\mathrm{W}$ lattice atom in order to produce a Frenkel pair. It seems reasonable that this transferred energy should be of the order of the vacancy formation enthalpy. In the presence of a sufficiently high solute D concentration, D can react with a produced vacancy before it recombines thus stabilizing it. Such a vacancy stabilization mechanism has already been proposed by Kato et al. [40] based on density functional theory calculations. The correspondingly produced interstitial atom is mobile at $300 \mathrm{~K}$ and can diffuse to a sink for interstitials (e.g. grain boundaries or surface). In this respect, the crucial step in this synergistic process is the stabilization of produced vacancies through reaction with solute D. By this vacancy stabilization the threshold for damage production could be much lower than the above cited values for displacement damage production of more than $40 \mathrm{eV}$.

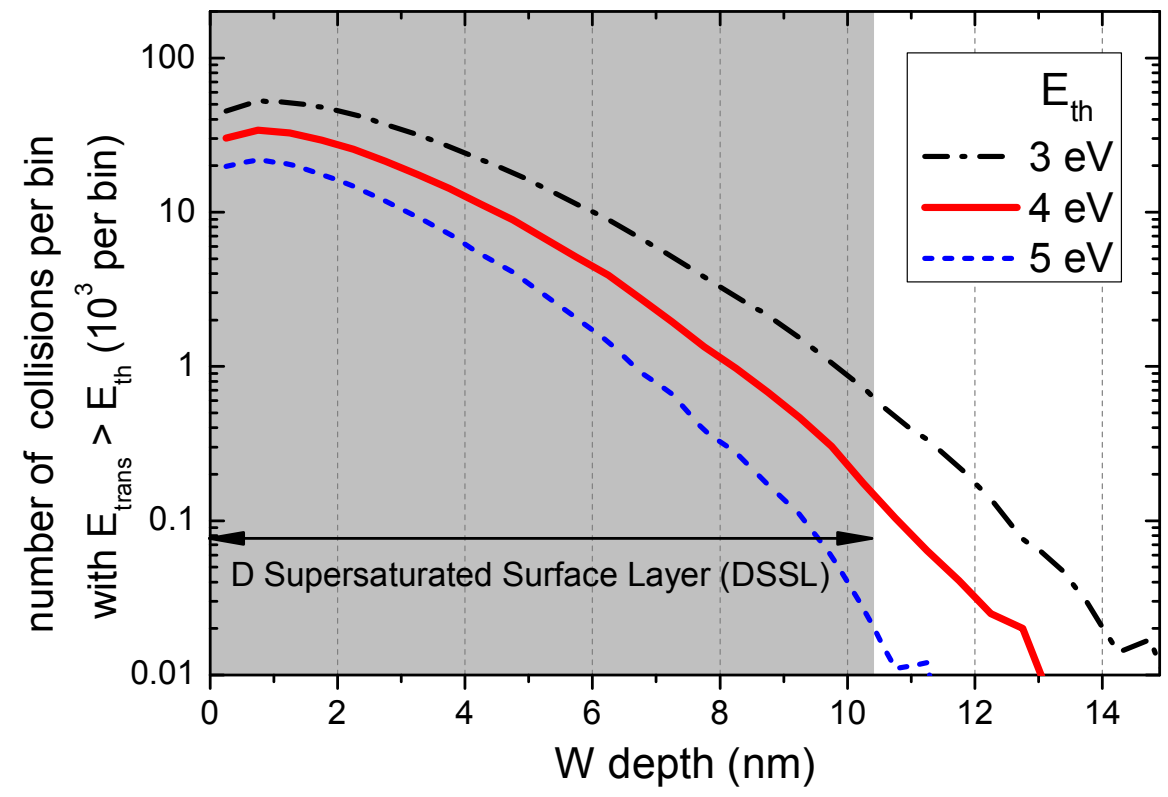

Figure 4. (color online only) SDTrimSP simulations of the number of collisions per depth interval (bin width $=0.5 \mathrm{~nm})$ with transferred energy above a certain threshold $(3 \mathrm{eV}, 4 \mathrm{eV}$ and $5 \mathrm{eV})$ as function of depth. The incident particle number is 20000 and the initial energy is $215 \mathrm{eV}$. The grey-shaded area marks the thickness of the DSSL.

To achieve a better understanding of the thickness of the DSSL, SDTrimSP simulations $[28,29]$ were carried out to determine the depth distribution of the energy transferred in D-W collisions. Results describing the case investigated here are plotted in Fig. 4. Shown is the energy transferred in $\mathrm{D}$ collisions with $\mathrm{W}$ lattice atoms as a function of depth applied for $\mathrm{D}^{+}$ ions with an initial energy of $215 \mathrm{eV}$. In total 20000 projectiles were simulated. Since this is not included in the standard SDTrimSP output the code was modified to save all calculated collision events. A 3-D spatially resolved probability distribution function (pdf) $p(E(x, y, z))$ of energy transferred from $\mathrm{D}$ atoms to $\mathrm{W}$ atoms is generated from the list of all simulated collision events. This three-dimensional pdf is subsequently integrated over the lateral coordinates $\mathrm{x}$ and $\mathrm{y}$ to obtain the one-dimensional probability function $\mathrm{p}(\mathrm{E}(\mathrm{z}))$, which now depends on the depth $\mathrm{z}$ only. From this distribution the respective fraction (or number) of collisions exceeding a given energy threshold value can be extracted. For atomic D impinging 
with an energy of $215 \mathrm{eV}$ the number of collisions at a depth of $10 \mathrm{~nm}$ with an energy transfer of more than $4 \mathrm{eV}$ to a W atom is already well below 0.005 times the number of corresponding D-W collisions in the first $1 \mathrm{~nm}$, and decays exponentially with further increasing depth. At the surface a $\mathrm{D}$ atom with energy of $215 \mathrm{eV}$ can at maximum transfer $9.2 \mathrm{eV}$. In a depth of $10 \mathrm{~nm}$, i.e. at the end of the DSSL, the transferable energy is much lower. For the molecular species with correspondingly lower energy per atom $(1 / 2$ or $1 / 3$ of the energy of the atomic species) the additional contributions at larger depth are negligible. In Fig. 4 we plot the number of collisions per depth interval with a transferred energy higher than a certain threshold energy $E_{\text {th }}$ for values of $E_{\text {th }}=3,4$ and $5 \mathrm{eV}$. If we consider a threshold of $1 \%$ of the peak value of the number of collisions per depth interval (occurring at a depth of about $1 \mathrm{~nm}$ ) as guideline to estimate the extend up to which collision effects can contribute, then the width of $10 \mathrm{~nm}$ of the DSSL can be used to estimate a value for $E_{\text {th. }}$ This yields a value of $E_{t h}=4 \pm 1 \mathrm{eV}$, corresponding to $93 \pm 23 \mathrm{eV} \mathrm{D}$ projectile energy. To check our postulate, we have actually exposed $\mathrm{W}$ samples to $\mathrm{D}$ plasma with ion energies ranging from 60 to $415 \mathrm{eV}$. SEM images showing the surfaces of these samples can be found in Fig. 5 . Above the postulated threshold energy, the whole sample surface is strongly distorted $(215 \mathrm{eV}$ (Fig. 5c) and $415 \mathrm{eV}$ (Fig. 5d)). Below the threshold energy, i.e. at $60 \mathrm{eV}$ (see Fig. 5a), no visible damage is observed for all the grains investigated. With an energy comparable to the predicted threshold $(\sim 115 \mathrm{eV}$, see Fig. $5 \mathrm{~b})$, one can clearly see that the surface is partially distorted, meaning that some grains are severely damaged and others look still pristine. The dependence of the damaging threshold on the grain orientations is presently not the focus and relevant experiments have been planned for the future.

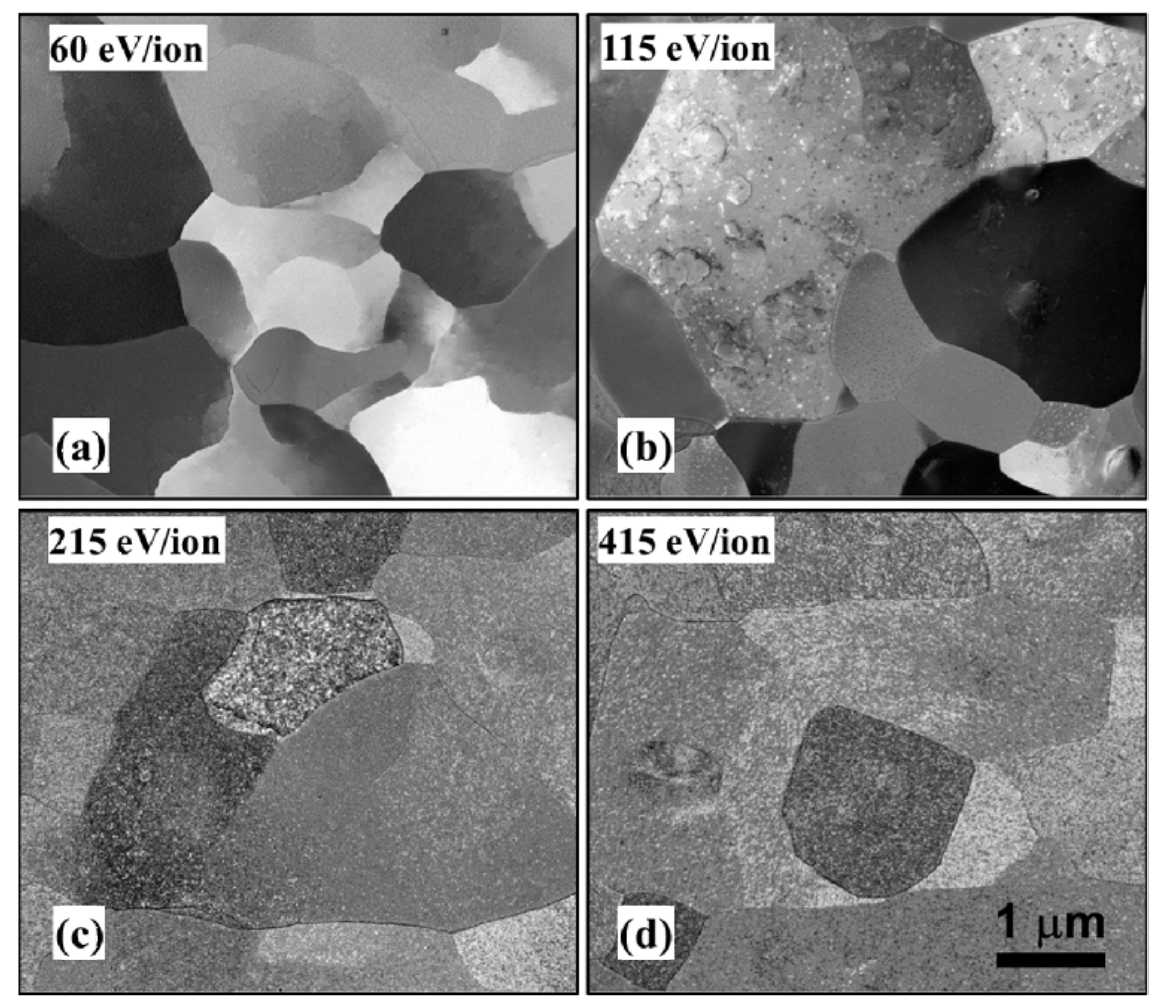

Figure 5. SEM images (produced using $5 \mathrm{keV}$ electrons) of $W$ surfaces after D implantation to fluence of $1 \times 10^{24} \mathrm{D} / \mathrm{m}^{2}$ at $300 \mathrm{~K}$ with different ion energies with respect to the predicted ion energy threshold of $93 \pm 23 \mathrm{eV}$ for DSSL formation: a) $60 \mathrm{eV/ion}$, which is below the threshold; b) $115 \mathrm{eV} / \mathrm{ion}$, which is within the predicted threshold energy range; c) $215 \mathrm{eV/ion}$ as for samples with Ar-sputter depth profiling; d) 415 eV/ion. 
The fact that the D concentration is rather homogenous throughout the whole DSSL thickness suggests that the damaging process is for our experimental conditions already strongly saturated. The estimated value of $E_{t h}$ is close to the formation enthalpy of thermodynamic vacancies, which is about $3.5 \mathrm{eV}$ [41-43]. Furthermore, it has been reported that solute $\mathrm{H}$ reduces the vacancy formation energy in $\mathrm{W}$ by up to $25 \%$ for extreme solute $\mathrm{H}$ concentrations [44] thus further facilitating formation of Frenkel pairs. In addition, vacancies appear to have an attractive interaction in the presence of solute $\mathrm{H}$ so that the clustering of vacancies into larger vacancy complexes seems possible [44, 45]. Eventually this postulated process could lead to the evolution of larger defect structures. Because isolated defects, such as single vacancies, are not sufficient to explain the blurring of the Kikuchi pattern and SEM images such a clustering provides a basis for explaining the experimental observations. Based on these considerations we assume that this new damage production process vanishes for $\mathrm{D}$ bombardment energies lower than about $100 \mathrm{eV}$.

We have argued above that damage can be induced by the synergistic interaction of solute and energetic $\mathrm{D}$ with the $\mathrm{W}$ lattice and be stabilized by interaction with $\mathrm{D}$. That triggers the question whether this damage will disappear if the D is released, e.g., after degassing. Samples after D implantation were annealed at different temperatures for different durations (see in Sec. 2.4). Previous studies [30, 31] showed that almost all D is released from the sample for temperatures above $750 \mathrm{~K}$. By NRA measurements we also confirmed that the remaining $\mathrm{D}$ amount in the sample after annealing at $900 \mathrm{~K}$ is below the detection limit. However, a lot of surface defects are still visible by SEM after annealing at $900 \mathrm{~K}$ for $10 \mathrm{~min}$ (Fig. 3e). It turned out that the D-induced surface distortion disappears only after annealing at $1200 \mathrm{~K}$ (Fig. 3f). The persistence of these defects after D degassing reveals that they are stable even without the presence of $\mathrm{D}$. Although a high $\mathrm{D}$ concentration is required to produce this surface distortion, such-produced defects do not disappear if the $\mathrm{D}$ is released. This points to the fact that the energy required for desorbing $\mathrm{D}$ from these defects is lower than that for annealing them. Naturally, it cannot be excluded that a minor part of the defects may have already been annealed at temperatures $\leq 900 \mathrm{~K}$ or they may further cluster at high temperatures.

\section{Conclusion}

The novel low-energy argon-sputter depth-profiling method with a high depth resolution $(\sim 3 \mathrm{~nm})$ allows us to resolve the 10-nm-thick DSSL with $9.4 \pm 0.7$ at. \% D concentration in W surfaces after irradiation with ion energy of $215 \mathrm{eV}$. Choosing shorter Ar-sputtering steps the achievable depth resolution can possibly be enhanced in future experiments. Found in almost all D-implanted W surfaces, the thin D-rich layer is confirmed and further resolved in our experiments. It is reasonable to assume that a similar $\mathrm{H}$-rich layer can be produced by $\mathrm{H}$ implantation. Such an H-rich layer could well be visualized by SEM and quantified with ${ }^{15} \mathrm{~N}$ NRA with high depth resolution. The latter was so far not attempted because the anticipated $\mathrm{H}$ concentration was significantly lower than the $10 \%$ found here. In addition, a high concentration of $\mathrm{H}$ at the surface might also have been attributed to $\mathrm{H}$-rich adsorbates such as water or hydrocarbons (e.g., from air exposure or residual gas). By using the rare hydrogen isotope D in our sputter depth profiling experiments, we were able to clearly show that the very large observed concentration of $\mathrm{H}$ isotopes is actually located below the $\mathrm{W}$ surface.

The unexpectedly high D concentration in the DSSL is attributed to a highly distorted W layer created during D implantation, as confirmed by EBSD measurements. We put forward 
the hypothesis that the strong lattice distortion is induced by the synergistic interaction of solute $\mathrm{D}$ and the impinging energetic $\mathrm{D}$ atoms with the $\mathrm{W}$ lattice. This process appears to have a substantially lower threshold than the creation of stable Frenkel pairs in W by a purely kinetic process. We suggest that the most important step in this interaction process is the stabilization of produced vacancies by interaction with solute $\mathrm{D}$ and there is indication of defect clustering leading to stable defect structures. In principle, the novel damaging mechanism active in the production of the DSSL in W surfaces could also occur for other Hirradiated metal surfaces. Furthermore, the proposed mechanism might have consequences for the evolution of neutron-induced lattice damage in future fusion devices. If stabilization of vacancies by reaction with solute $\mathrm{H}$ isotopes is also active in that case it is possible that the accumulation of defects in the presence of a significant solute $\mathrm{H}$ concentration proceeds more efficiently than in the absence of $\mathrm{H}$ and thus the defect density and as a consequence the hydrogen fuel retention may reach higher saturation levels.

\section{Acknowledgements}

The stay of L. Gao at Max-Planck Institute for Plasma Physics in Garching (Germany) was partially funded through a Joint Doctoral Promotion Program between Max-Planck Society and the Chinese Academy of Sciences, which is gratefully acknowledged. Fruitful discussion with Matej Mayer on the interpretation of results from ion beam analysis is highly appreciated. We are also grateful to Karsten Schlüter and Gabriele Matern for help on EBSD and SEM imaging. Thanks are further due to Michael Fußeder and Joachim Dorner for help with the NRA measurements.

\section{APPENDIX: Estimate of the Solute D Concentration}

Assume a tungsten sample of thickness $d$ at temperature $T$ and with an interstitial diffusion coefficient for deuterium of $D(T)$, which we take as the value determined for $\mathrm{H}$ in $\mathrm{W}$ by Frauenfelder [36] divided by $\sqrt{2}$. Let the reflection coefficient of incident $\mathrm{D}$ ions be $R(E)$, where $E$ is the kinetic energy of the ions, and $r_{\text {impl }}(E)$ the implantation range of the ions. For simplicity, we assume in a first step that the stopping profile of the $\mathrm{D}$ ions is a delta function at $r_{\text {impl }}(E)$.

We now look at the system in steady state, where the deuterium diffusing through the sample has already reached the rear surface of the sample at $x=d$ (the implanted surface is at $x=0$ ) and the permeation flux has become constant. The boundary condition at either surface is assumed to be that the solute deuterium concentration $c=0$, i.e. the emission of $\mathrm{D}$ through either surface is diffusion limited. In the review by Causey et al. [10], it is suggested that this may indeed be a reasonable assumption for $\mathrm{H}$ isotopes in W. Solution of the diffusion equation then shows that two linear concentration gradients $\partial c / \partial x$ have built up from either surface with $c=0$ to $x=r_{\text {impl }}(E)$. Here the concentration reaches its peak value, which we call $c_{\max }$. Towards the implantation surface, the concentration gradient is

$$
\partial c / \partial x=c_{\max } / r_{\text {impl }}
$$

and towards the back surface it is

$$
\partial c / \partial x=c_{\max } /\left(d-r_{i m p l)} \approx^{c_{\max }} / d\right.
$$


For the implantation conditions we used here, the implantation range $r_{i m p l}(E)$ is of the order of $10 \mathrm{~nm}$ (see Fig. 4). Our sample thickness is $\sim 700 \mu \mathrm{m}$. Because for our temperatures and plasma exposure times the diffusing deuterium does not reach the back surface, we can make another approximation: From NRA depth profiling we know that by the end of our plasma exposure, we find trapped deuterium up to a depth of several $\mu \mathrm{m}$ [17-20]. Simulations with a numerical diffusion-trapping code $[19,46]$ show that the traps in the sample act as a highly effective sink for deuterium, which leads to a relatively sharp boundary between $\mathrm{W}$ with filled and $\mathrm{W}$ with empty traps, which propagates into the sample with increasing time and fluence. At this boundary, the solute concentration is much smaller than $c_{\max }$ at the implantation range, i.e. $c \approx 0$. Accordingly, for our further considerations, let us set $d>1 \mu \mathrm{m}$, and therefore $d>r_{\text {impl }}(E)$.

According to Fick's law, the diffusion flux $j$ is given by

$$
j=D(T) \cdot \rho_{W} \cdot \partial c / \partial x
$$

where $\rho_{W}$ is the atom density of tungsten. Consequently, the diffusion flux $j_{b u l k}$ towards $x=d$ is much smaller than the re-emission flux $j_{\text {surface }}$ back to the plasma-exposed $\mathrm{W}$ surface. Since we assumed steady state, the implantation flux $j_{\text {impl }}$, which is given by $(1-R(E)) \cdot j_{\text {incident }}$, is balanced by the sum of $j_{\text {bulk }}$ and $j_{\text {surface }}$. By neglecting the small contribution $j_{\text {bulk }}$ we arrive at

$$
(1-R(E)) \cdot j_{\text {incident }}=j_{\text {bulk }}+j_{\text {surface }} \approx j_{\text {surface }}
$$

Based on this approximation we can calculate the peak concentration $c_{\max }$ at $r_{\text {impl }}$ :

$$
C_{\max }=\frac{(1-R(E)) \cdot j_{\text {incident }} \cdot r_{\text {impl }}}{\rho_{W} \cdot D(T)}
$$

If we compare this simple analytic estimate to calculations with a numerical diffusiontrapping model $[19,46]$ that includes the full deposition profile of implanted D ions, we see that the values for $c_{\max }$ are in excellent agreement if $r_{i m p l}$ is replaced by the mean range of the ions, $r_{\text {mean }}$, and that the actual maximum concentration reaches about $90 \%$ of the stationary value $c_{\max }$ within a few seconds.

Based on the implantation profiles calculated by SDTrimSP [28, 29] (as shown in Fig. 4) for the exposure conditions used here, we obtain $r_{\text {mean }}=3.9 \mathrm{~nm}$. The reflection coefficient is $R=$ 0.69 and is derived as the weighted average of the reflection coefficients for $\mathrm{D}^{+}, \mathrm{D}_{2}{ }^{+}$and $\mathrm{D}_{3}{ }^{+}$, which are taken from Equation (6) in [47]. The incident total deuteron flux is $j_{\text {incident }}=9.9 \times 10^{19} \mathrm{D} \mathrm{m}^{-2} \mathrm{~s}^{-1}$, and for $T=300 \mathrm{~K}$ the diffusion coefficient for deuterium is $D=8.4 \times 10^{-14} \mathrm{~m}^{2} \mathrm{~s}^{-1}$ according to Frauenfelder's expression [36] divided by $\sqrt{2}$. Using finally $\rho_{W}=6.2 \times 10^{28}$ atoms $/ \mathrm{m}^{3}$, we arrive at $c_{\max }=2.3 \times 10^{-3}$ at. $\%$ according to Eq. (A5). This is almost four orders of magnitude lower than the determined concentration for trapped $\mathrm{D}$ in the DSSL. By this, we suppose there is a new damaging process being responsible for the formation of the DSSL. 


\section{References:}

[1] L. Schlapbach, A. Züttel. Hydrogen-storage materials for mobile applications, Nature 414, 353-358 (2001).

[2] D.K. Ross. Hydrogen storage: The major technological barrier to the development of hydrogen fuel cell cars, Vacuum 80, 1084-1089 (2006).

[3] S.G. Chalk, J.E. Miller. Key challenges and recent progress in batteries, fuel cells, and hydrogen storage for clean energy systems, J Power Sources 159, 73-80 (2006).

[4] C. D. Beachem. A new model for hydrogen-assisted cracking (hydrogen "embrittlement"). Metall. Trans. 3, 441-455 (1971).

[5] H.K. Birnbaum, P. Sofronis. Hydrogen-enhanced localized plasticity-a mechanism for hydrogenrelated fracture, Mater. Sci. Eng. A 176, 191-202 (1994).

[6] A. Pundt, R. Kirchheim. HYDROGEN IN METALS: Microstructural Aspects, Ann. Rev. Mater. Res. 36, 555-608 (2006).

[7] S.M. Myers, M.I. Baskes, H.K. Birnbaum, J.W. Corbett, G.G. DeLeo, S.K. Estreicher, E.E. Haller, P. Jena, N.M. Johnson, R. Kirchheim, S.J. Pearton, M.J. Stavola. Hydrogen interactions with defects in crystalline solids, Rev. Mod. Phys. 64, 559-617 (1992).

[8] T. Hirai, F. Escourbiac, S. Carpentier-Chouchana, A. Fedosov, L. Ferrand, T. Jokinen, V. Komarov, A. Kukushkin, M. Merola, R. Mitteau, R.A. Pitts, W. Shu, M. Sugihara, B. Riccardi, S. Suzuki, R. Villari. ITER tungsten divertor design development and qualification program, Fusion Eng. Des. 88, 1798-1801 (2013).

[9] R.A. Pitts, S. Carpentier, F. Escourbiac, T. Hirai, V. Komarov, S. Lisgo, A.S. Kukushkin, A. Loarte, M. Merola, A. Sashala Naik, R. Mitteau, M. Sugihara, B. Bazylev, P.C. Stangeby. A full tungsten divertor for ITER: Physics issues and design status, J. Nucl. Mater. 438, Supplement S48-S56 (2013).

[10] R.A. Causey. Hydrogen isotope retention and recycling in fusion reactor plasma-facing components, J. Nucl. Mater. 300 , 91-117 (2002).

[11] J. Roth, K. Schmid. Hydrogen in tungsten as plasma-facing material, Phys. Scripta. T145, 014031 (2011).

[12] Y. Hatano, M. Shimada, T. Otsuka, Y. Oya, V.Kh. Alimov, M. Hara, J. Shi, M. Kobayashi, T. Oda, G. Cao. Deuterium trapping at defects created with neutron and ion irradiations in tungsten. Nucl. Fusion, 53, 073006 (2013)

[13] E. Markina, M. Mayer, A. Manhard, and T. Schwarz-Selinger. Recovery Temperatures of Defects in Tungsten Created by Self-implantation. J. Nucl. Mater. 463, 329-332 (2015).

[14] M. Wilde, K. Fukutani. Hydrogen detection near surfaces and shallow interfaces with resonant nuclear reaction analysis, Surf. Sci. Rep. 69, 196-295 (2014).

[15] V.K. Alimov, M. Mayer, J. Roth. Differential cross-section of the D $\left({ }^{3} \mathrm{He}, \mathrm{p}\right){ }^{4} \mathrm{He}$ nuclear reaction and depth profiling of deuterium up to large depths, Nucl. Instr. Meth. Phys. B 234, 169-175 (2005).

[16] K. Schmid, U. von Toussaint. Statistically sound evaluation of trace element depth profiles by ion beam analysis, Nucl. Instr. Meth. Phys. B 281, 64-71 (2012).

[17] P. Wang, W. Jacob, L. Gao, T. Dürbeck, T. Schwarz-Selinger. Comparing deuterium retention in tungsten films measured by temperature programmed desorption and nuclear reaction analysis, Nucl. Instr. Meth. Phys. B 300, 54-61 (2013).

[18] K.A. Moshkunov, K. Schmid. Tungsten bending under hydrogen irradiation, J. Nucl. Mater. 424, 89-93 (2012). 
[19] K. Schmid, V. Rieger, A. Manhard. Comparison of hydrogen retention in W and W/Ta alloys, J. Nucl. Mater. 426, 247-253 (2012).

[20] M. Balden, A. Manhard, S. Elgeti. Deuterium retention and morphological modifications of the surface in five grades of tungsten after deuterium plasma exposure, J. Nucl. Mater. 452, 248-256 (2014).

[21] L. Gao, W. Jacob, T. Schwarz-Selinger, A. Manhard. Deuterium implantation into tungsten nitride: Negligible diffusion at 300 K, J. Nucl. Mater. 451, 352-355 (2014).

[22] L. Gao, W. Jacob, G. Meisl, T. Schwarz-Selinger, T. Höschen, U.v. Toussaint, T. Dürbeck. Interaction of deuterium plasma with sputter-deposited tungsten nitride films, Nucl. Fusion 56, 016004 (2016).

[23] A. Manhard, G. Matern, M. Balden. A step-by-step analysis of the polishing process for tungsten specimens, Practical Metallography 50, 5-16 (2013).

[24] A. Manhard, T. Schwarz-Selinger, W. Jacob. Quantification of the deuterium ion fluxes from a plasma source, Plasma Sources Sci. T. 20, 015010 (2011).

[25] M. Mayer. SIMNRA User's Guide. Max-Planck-Institut für Plasmaphysik, IPP Report:IPP 9/113 (1997). (http://hdl.handle.net/11858/00-001M-0000-0027-6157-F)

[26] M. Mayer, E. Gauthier, K. Sugiyama, U. von Toussaint. Quantitative depth profiling of deuterium up to very large depths, Nucl. Instr. Meth. Phys. B 267, 506-512 (2009).

[27] P. Wang, W. Jacob, L. Gao, S. Elgeti, M. Balden. Deuterium retention in tungsten films deposited by magnetron sputtering, Phys. Scripta. T159, 014046 (2014).

[28] W. Möller, W. Eckstein, J.P. Biersack. Tridyn-binary collision simulation of atomic collisions and dynamic composition changes in solids, Comput. Phys. Commun. 51 , 355-368 (1988).

[29] A. Mutzke, R. Schneider R, W. Eckstein, R. Dohmen. IPP Report 12/08, Max-Planck-Institut für Plasmaphysik (Hrsg.) (2011) (http://hdl.handle.net/11858/00-001M-0000-0026-EAF9-A).

[30] A. Manhard, U.v. Toussaint, T. Dürbeck, K. Schmid, W. Jacob. Statistical analysis of blister bursts during temperature-programmed desorption of deuterium-implanted polycrystalline tungsten, Phys. Scripta. T145, 014038 (2011).

[31] A. Manhard, K. Schmid, M. Balden, W. Jacob. Influence of the microstructure on the deuterium retention in tungsten, J. Nucl. Mater. 415, S632-S635 (2011).

[32] P.W. Tamm, L.D. Schmidt. Binding States of Hydrogen on Tungsten, J. Chem. Phys. 54, 47754787 (1971).

[33] S. Markelj, O.V. Ogorodnikova, P. Pelicon, T. Schwarz-Selinger, I. Cadez. Temperature dependence of D atom adsorption on polycrystalline tungsten, Appl. Surf. Sci. 282, 478-486 (2013).

[34] O.K. Brice, B.L. Doyle. Steady state hydrogen transport in solids exposed to fusion reactor plasmas: Part I: Theory, J. Nucl. Mater. 120 , 230-244 (1984).

[35] Y. Ueda, H.T. Lee, H.Y. Peng, Y. Ohtsuka. Deuterium permeation in tungsten by mixed ion irradiation, Fusion Eng. Des. 87, 1356-1362 (2012).

[36] R. Frauenfelder. Solution and Diffusion of Hydrogen in Tungsten, J. Vac. Sci. Technol. 6, 388397 (1969).

[37] A. Manhard, M. Balden, S. Elgeti. Quantitative Microstructure and Defect Density Analysis of Polycrystalline Tungsten Reference Samples after Different Heat Treatments, Practical Metallography 52, 437-466 (2015).

[38] F. Maury, M. Biget, P. Vajda, A. Lucasson, P. Lucasson. Frenkel pair creation and stage I recovery in W crystals irradiated near threshold, Radiation Effects 38, 53-65 (1978). 
[39] K. Nordlund, A. E. Sand, F. Granberg, S. J. Zinkle, R. Stoller, R. S. Averback, T. Suzudo, L. Malerba, F. Banhart, W. J. Weber, F. Willaime, S, Dudarev, D. Simeone. Primary radiation damage in materials, Nuclear Science Report NEA/NSC/DOC (2015) 9 (OECD Nuclear Energy Agency, (https://www.oecd-nea.org/science/docs/2015/nsc-doc2015-9.pdf).

[40] D. Kato, H. Iwakiri, Y. Watanabe, K. Morishita, T. Muroga. Super-saturated hydrogen effects on radiation damages in tungsten under the high-flux divertor plasma irradiation, Nucl. Fusion 55, 083019 (2015).

[41] K.D. Rasch, R.W. Siegel, H. Schultz. Quenching and recovery investigations of vacancies in tungsten, Philosophical Magazine A 41, 91-117 (1980).

[42] D. Nguyen-Manh, A.P. Horsfield, S.L. Dudarev. Self-interstitial atom defects in bec transition metals: Group-specific trends, Phys. Rev. B 73, 020101 (2006).

[43] K. Heinola, T. Ahlgren, K. Nordlund, J. Keinonen. Hydrogen interaction with point defects in tungsten. Phys. Rev. B 82, 094102(2010).

[44] S.C. Middleburgh, R.E. Voskoboinikov, M.C. Guenette, D.P. Riley. Hydrogen induced vacancy formation in tungsten, J. Nucl. Mater. 448, 270-275 (2014).

[45] Y. Fukai. Formation of superabundant vacancies in $\mathrm{M}-\mathrm{H}$ alloys and some of its consequences: a review, J. Alloy. Compd. 356-357, 263-269 (2003).

[46] K. Schmid, U. von Toussaint, T. Schwarz-Selinger. Transport of hydrogen in metals with occupancy dependent trap energies. J. Appl. Phys. 116, 11 (2014).

[47] W. Eckstein, Reflection (Backscattering). Max-Planck-Institut für Plasmaphysik, IPP Report 17/12 (2009) (http://hdl.handle.net/11858/00-001M-0000-0026-F340-E). 